\title{
CVs Around the Minimum Orbital Period
}

\author{
S. Zharikov ${ }^{1}$, G. Tovmassian ${ }^{1}$ \\ ${ }^{1}$ Observatorio Astronomico Nacional, Instituto de Astronomia, Universidad Nacional Autonoma de Mexico, Ensenada, \\ BC, Mexico, 22860
}

Corresponding author: zhar@astrosen.unam.mx

\begin{abstract}
We discussed features of Cataclysmic Variables at the period minimum. In general, most of them must be WZ Sge-type objects. Main characteristics of the prototype star (WZ Sge) are discussed. A part of WZ Sge-type objects has evolved past the period limit and formed the bounce back systems. We also explore conditions and structure of accretion disks in such systems. We show that the accretion disk in a system with extreme mass ratio grows in size reaching a 2:1 resonance radius and are relatively cool. They also become largely optically thin in the continuum, contributing to the total flux less than the stellar components of the system. In contrast, the viscosity and the temperature in spiral arms formed at the outer edge of the disk are higher and their contribution in continuum plays an increasingly important role. We model such disks and generate light curves which successfully simulate the observed double-humped light curves in the quiescence.
\end{abstract}

Keywords: cataclysmic variables - dwarf novae - period minimum.

\section{Introduction}

A widely accepted evolutionary theory of cataclysmic variables (CVs), as presented in Kolb \& Baraffe (1999, and references therein), predicts a significant accumulation of CV systems around the orbital period minimum (Paczynski 1981). It also envisions that $\sim 70 \%$ of the current CV's population has evolved past the orbital period minimum and formed so-called bounce-back systems.

Figure 1 illustrates the current concept of CV evolution at the orbital period turn-around point on the mass-transfer rate and mass-ratio to orbital period diagrams. Cataclysmic Variables with orbital periods close to the 80 min orbital period minimum that undergo infrequent (years to decades) super-outbursts are called WZ Sge-type stars. Objects with short periods that have not been observed in outburst or super-outburst but have spectral characteristics similar to WZ Sge are listed also as WZ Sge-type candidates. Those systems are dominated at the period minimum.

The bounce-back systems are Cataclysmic Variables evolved beyond the minimum period limit, which is reached when the secondary star becomes of a substellar mass (brown dwarf) and partially degenerate. Bounce-back systems are expected to float within the 80 - 100 min orbital period range. Bounce- back systems are spectroscopically similar to these, but not every WZ Sge-type object has necessarily passed through the turning point. Figure 1 also displays the expected position of the bounce-back systems.

In this paper we discuss observational characteristics of cataclysmic variables at the period minimum and explore conditions and structure of its accretion disk.

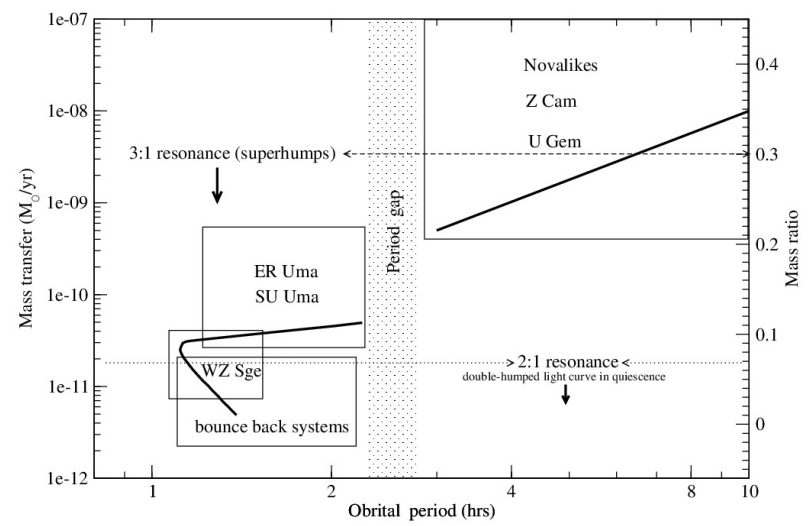

Figure 1: Schematic distribution of CV-types on the plot of the mass ratio and mass transfer rate vs. the orbital period.

\section{WZ Sge-Type Stars}

WZ Sge is the prototype star of the class of short-period cataclysmic variables (named WZ Sge-type stars). It is a high inclination $\left(\sim 77^{\circ}\right.$; Steeghs et al. 2007) cataclysmic variable with a late M-type dwarf secondary star $\left(0.078 M_{\odot}<M_{2}<0.13 M_{\odot}\right)$ orbiting at $81.6 \mathrm{~min}$ the fastest-spinning ( $28 \mathrm{sec}$, Robinson et al 1978) 
white dwarf $\left(0.88 M_{\odot}<M_{1}<1.53 M_{\odot}\right)$. The distance to WZ Sge is only $43.5 \mathrm{pc}$. For most of its life it is in quiescence with a $V \sim 15$, corresponding to $M_{V} \sim 12$. Below the main characteristics of WZ Sge are summarised:

- a short orbital period of $81.6 \mathrm{~min}$, close to the predicted period minimum of CVs $\sim 77$ min with a main sequence secondary.

- a spectrum in quiescence shows strong double-peaked Balmer emission lines from the accretion disk surrounded by broad absorptions, formed by the primary white dwarf (see for an example Howell et al. (2008)).

- an infrequent $\sim 20-30 y r$ and largeamplitude ( 8mag; 1913, 1946, 1978, 2001 yy.) super-outbursts succeeded by echo outbursts, absence of normal (as in SU Umatype stars) outbursts. In order to avoid an earlier occurrence of normal outburst in WZ Sge-type systems, it can be accepted as an extreme low viscosity parameter $a \sim 0.01$ 0.001 in accretion disks (Smak (1993), Osaki (1994)).

- The light curve during a super-outburst shows long-lasting super-humps (Patterson, et al. 2002).

- The optical light curve are double-humped sometimes during super-outburst and in quiescence (Patterson, et al. 1998; Patterson, et al. 2002).

- There is evidence of forming spiral arms in the disk during super-outburst (Baba et al. 2002, Howell et al. 2003).

- In quiescence the accretion disk is asymmetric, and the bright spot region is shown to be extended along the mass transfer stream (Skidmore et al. 2000, Mason et al. 2000).

- The outer layers of the accretion disk must be of low density and low temperature $\sim 3000 \mathrm{~K}$ (Howell et al. 2004).

- A cavity most likely formed in the inner part of the disk during quiescence implying an annulus-shaped accretion disk (Kuulkers et al. 2011).

- The outer radius of the disk is about a $3: 1$ resonance radius $\left(r_{\text {disk }} \leq R_{3: 1}\right)$ in quiescence and it can reach to $2: 1$ resonance radius $\left(r_{d i s k} \leq R_{2: 1}\right)$ during a super-outburst.

There are about $\sim 100$ objects proposed or confirmed as WZ Sge-type systems. Most of them were suggested based on features of spectra or the amplitude of the super-outbursts. Figure 2 summarise current conception about WZ Sge-type systems which do not yet pass the period minimum.

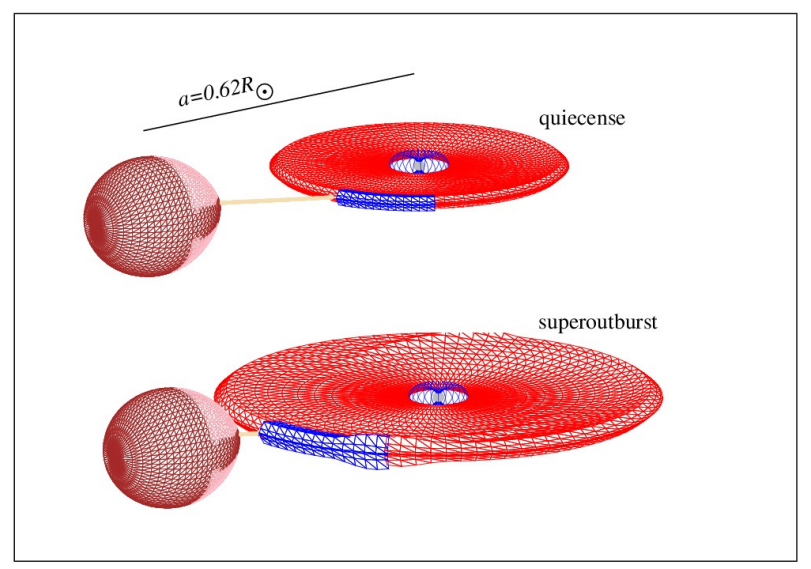

Figure 2: The current conception about WZ Sge- type systems which do not yet pass yet the period minimum.

\section{Bounce-Back Systems}

After reaching the period minimum the CVs should evolve back toward longer periods and form a so-called bounce-back or post-period minimum systems. The increasing of orbital periods of bounce-back systems is accompanied by a decline in mass transfer rate about an order of magnitude according to the existing models of CVs evolution (Kolb \& Barrafe 1999, Sirotkin \& Kim 2010). The list of the bounce back candidates are given in Table 2 and Figure 3 (Zharikov et al. 2013). The optical spectra in quiescence of proposed candidates are similar to WZ Sge. All those systems include a massive and relatively cool $(\sim 12000 K)$ white dwarf primary and a $M_{2} / M_{1}$ mass ratio less than 0.075 . Such low mass ratio implies a Jupiter- size secondary (a late type M-dwarf or a brown dwarf) and with a 2:1 resonance radius is within of the Roche lobe of the primary. Also, similar to WZ Sge, the bounce back candidates do not show normal outbursts which implies an extremely low $\alpha \sim 0.01-0.001$ viscosity parameter (Smak (1993), Osaki (1994)). This together with the low $\sim 10^{-11} M_{\odot} /$ year mass transfer rate, allows the accretion disk to expand in bounce back systems up to a 2:1 resonance radius and form a two spiral wave structure in those systems (Lin \& Papaloizou, 1979).

The large size of the disk and domination of white dwarf radiation in the optical range and the secondarys radiation in the $J H K$ bands in SDSS0804 and SDSS1238 bounce back candidates (Zharikov et al 2013, Aviles et al 2010) lead to the conclusion that the "standard accretion disk model" (Frank et al. 2002) does not apply to bounce-back systems. 
CVs Around the Minimum Orbital Period

Table 1: Parameters of WZ Sge and of bounce-back candidates.

\begin{tabular}{l|lccccccc}
\hline NN/Object & $\begin{array}{l}\mathrm{P}_{\text {orb }} \\
(\text { days })\end{array}$ & $\begin{array}{c}\mathrm{V} \\
(\mathrm{mag})\end{array}$ & $\mathrm{q}$ & $\begin{array}{c}\mathrm{M}_{1} \\
\left(\mathrm{M}_{\odot}\right)\end{array}$ & $\begin{array}{c}\mathrm{M}_{2} \\
\left(\mathrm{M}_{\odot}\right)\end{array}$ & $\begin{array}{c}\mathrm{T}_{\text {eff }}^{W D}(K) \\
(K)\end{array}$ & $\begin{array}{c}i \\
\left({ }^{o}\right)\end{array}$ & $\mathrm{LC}^{1}$ \\
\hline WZ Sge & 0.0567 & $\sim 15$ & 0.092 & 0.85 & 0.078 & 13500 & 77 & $+\mathrm{sq}$ \\
1. GW Lib* & 0.0533 & 19.1 & 0.060 & 0.84 & 0.05 & & 11 & \\
\hline 2. V455 And & 0.0563 & 16.5 & $0.060 \mathrm{~s}$ & & $>\mathrm{M} 9$ & 11500 & 83 & $+\mathrm{q}$ \\
3. AL Com* & 0.0567 & 19.1 & 0.060 & & & 16300 & & $+\mathrm{q}$ \\
4. SDSS1035 & 0.057 & 18.7 & $0.055 \mathrm{e}$ & 0.94 & 0.05 & 10100 & 83 & \\
5. SDSS1238 & 0.056 & 17.8 & 0.05 & $\sim 1.0$ & 0.05 & 12000 & $\sim 70$ & $+\mathrm{q}$ \\
6. SDSS0804* & 0.059 & 17.8 & $0.05 \mathrm{~s}$ & $\sim 0.9$ & 0.045 & 13000 & $\sim 70$ & $+\mathrm{q}$ \\
7. EG Cnc* & 0.060 & 18.8 & $0.035 \mathrm{~s}$ & & & 12300 & & $+\mathrm{s}$ \\
8. RX1050-14 & 0.062 & 17.6 & $<0.055 \mathrm{v}$ & & & 13000 & $<65$ & \\
9. GD552 & 0.0713 & 16.6 & $<0.052 \mathrm{v}$ & & $<0.08$ & 10900 & $<60$ & \\
10. RE1255 & 0.083 & 19.0 & $<0.064 \mathrm{v}$ & $>0.9$ & $<0.08$ & 12000 & $<5$ & - \\
\hline
\end{tabular}

${ }^{1}$ light curve (LC) features: " +" LC shows a double-hump during the orbital period; "s" - during super-outburst; "q" - during quiescence; "-" absent of double-humps in LC. * - objects which demonstrated WZ Sge-type superoutburst.

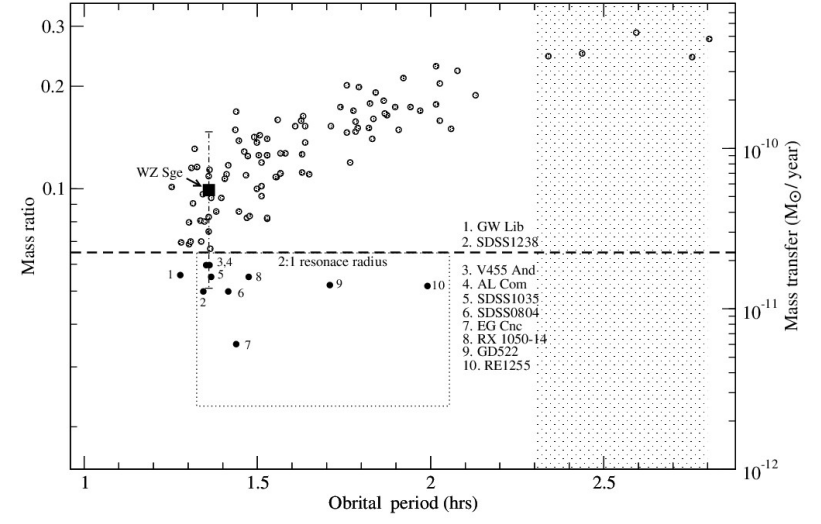

Figure 3: The systems with known mass ratio vs. orbital period are plotted. The left axes is the mass ratio and estimated mass transfer rates are given on the right axes. The bounce-back candidates are enclosed in a dash-dotted box.

Cannizzo \& Wheeler (1984) studied the vertical structure of a steady-state, $\alpha$-model thin-accretion disk for an accreting object of $1 \mathrm{M}_{\odot}$. They found that, for low accretion rates, the disk structure is optically thin and can be double- valued with high- $(\sim 5000 \mathrm{~K})$ and low- $(\sim 2000 \mathrm{~K})$ temperature branches. For $\alpha>0.1$ a warm solution is possible in the inner region of the accretion disk, but disk annuli at larger radii will be in a cold state with $T<2000 \mathrm{~K}$. Only the low-temperature solution exists for $\alpha \approx 0.1$. As $\alpha$ decreases with temper- ature, the tendency to develop cold solutions in quiescence is enhanced. Therefore, accretion disks in bounce back systems are most probably cool $(\sim 2500 \mathrm{~K})$.

The optical light curve of the high inclination bounce back candidates shows permanently a doublehumped light curve (marked by " $+\mathrm{q}$ " in Table 2). The spiral arm structures were found from Doppler tomography mapping in quiescence in two well studied example of bounce back candidates SDSS1238 (Aviles et al. 2010) and SDSS0804 (Zharikov et al. 2013). Also important, that, because the mass transfer rate significantly decrease after the period minimum the size of the magnetosphere of the primary with faint magnetic field will increase with the decreasing of $\dot{M}$ and a relatively faint ( $\leq 1 \mathrm{MGauss}$ ) magnetic field is enough to form a cavity in the inner part of the accretion disk (Zharikov et al 2013).

Taking into account all these features a geometrical model of bounce back system was constructed to explain the observed double-humped light curve in quiescence. The model takes into account the positions of the bright structures in the Doppler maps, the large size of the accretion disk, and the description of the spiral density waves in Hachisu et al. (2004). Figure 4 presents the geometry used in the model (central panel) and grayscale images present the height of the disk (left) and the temperature distribution (right) of the accretion disk. 

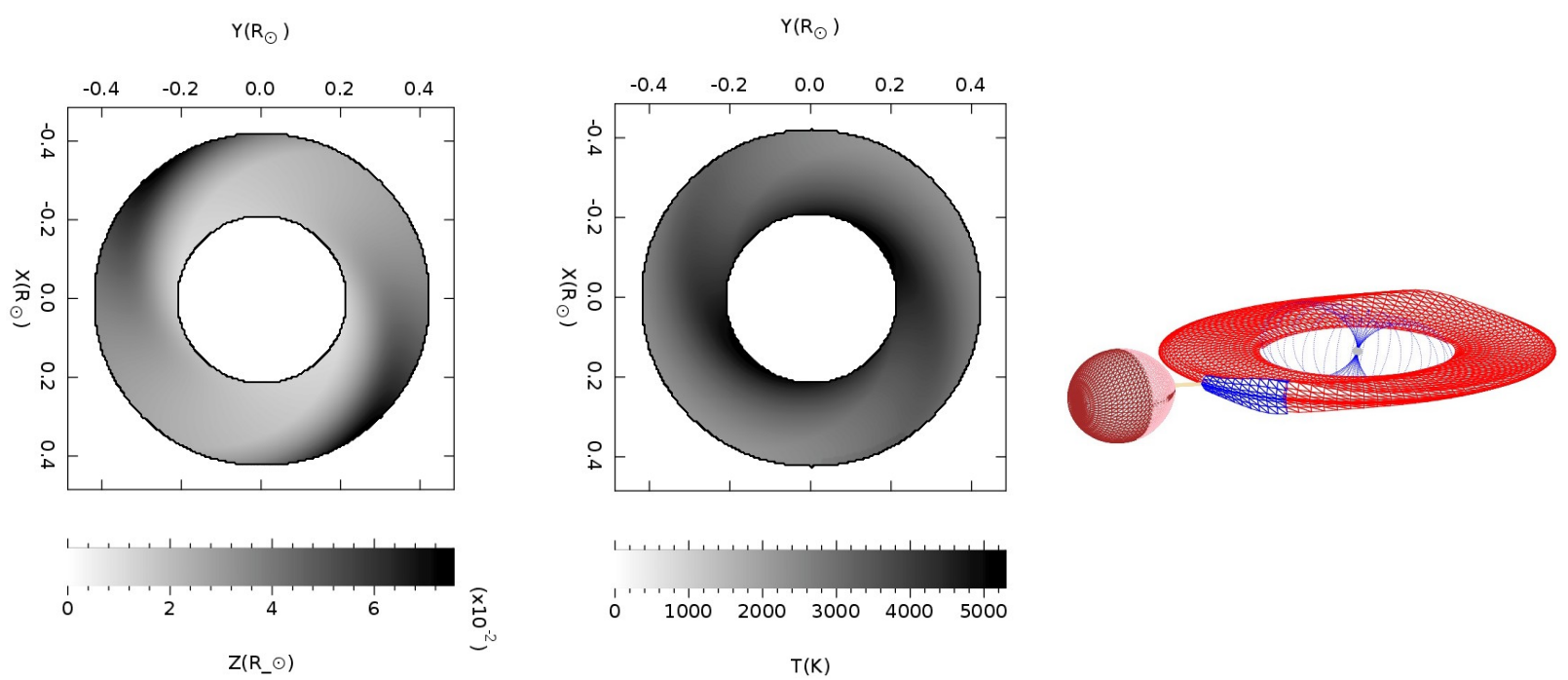

Figure 4: From left to right: vertical thickness of the accretion disk, model configuration used to calculate the light curves of bounce-back systems and the temperature distribution of the model.
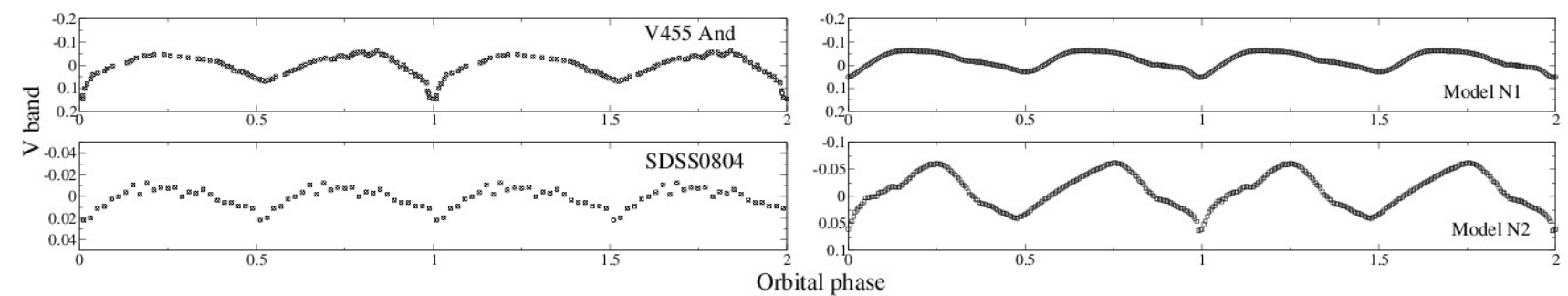

Figure 5: Left: examples of double-humped light curve; right: examples of the light curve generated by the model.

We calculated a variety of models using the typical average parameters of bounce-back systems presented in Table 1 and the double-hump-shape light curve (Fig. 5, (left panels) is easily reproduced by such models, two examples of which are shown in Fig. 5 , (right panels).

\section{Conclusions}

The galactic age is high enough that $\sim 70 \%$ of $\mathrm{CVs}$ will have reached the CVs orbital period minimum. In general, most of them must be WZ Sge-type objects. A part of WZ Sge-type objects have already passed the period limit and formed the bounce back systems. The structure of the disk must be changed when the system pass through the minimum. Before the minimum, mass transfer rate is higher and the accretion disk is hotter and smaller. After the period limit it is larger and reaches a 2:1 resonance radius. The disk is cool $(\sim$ $2500 \mathrm{~K}$ ) and has a cavity in the inner part and presents permanently existing spiral arms. The spirals should be denser and hotter that the rest of the disk. Based on these features we propose the double-humped light curve in quiescence state of a system together with orbital period longer than the period limits and the mass ratio of $q<0.08$ as the main manifestation of a WZ-Sge system which already passed through the minimum orbital period. When accretion has ended the CVs can form very close short period WD+BD systems, two examples of which are found recently (WD0137-349, Burleigh et al. (2006) and NLTT5306, Steele et al. (2013)).

\section{Acknowledgement}

We acknowledge PAPIIT grants IN-109209/IN-103912 and CONACyT grants 34521-E; 151858.

\section{References}

[1] Aviles, A., et al. 2010, ApJ, 711, 389

[2] Baba, H. et al. 2002, PASJ, 54, L7 
[3] Burleigh, M. R., et al. 2006, MNRAS, 373, L55 doi:10.1111/j.1745-3933.2006.00242.x

[4] Cannizzo, J. K., \& Wheeler, J. C. 1984, ApJS, 55, 367

[5] Frank, J., et al. 2002, Accretion Power in Astrophysics, Cambridge University Press doi:10.1017/CB09781139164245

[6] Hachisu, I. et al. 2004, APJL, 606, L139 doi:10.1086/421295

[7] Howell, S. B., et al. 2003, A\&A, 399, 219

[8] Howell, S. B., et al. 2004, ApJL, 602, L49 doi:10.1086/382481

[9] Howell, S. B., et al. 2008, ApJ, 685, 418 doi:10.1086/590491

[10] Kolb, U., \& Baraffe, I. 1999, MNRAS, 309, 1034

[11] Kuulkers, E., et al. 2011, A\&A, 528, A152

[12] Lin, D. \& Papaloizou, J., 1979, MNRAS, 186, 799 doi:10.1093/mnras/186.4.799

[13] Osaki, Y. 1995, PASJ, 47, 47

[14] Mason, E., et al. 2000, MNRAS, 318, 440

[15] Paczynski, B. 1981, Acta Astron., 31, 1

[16] Patterson, J., et al 1998, PASP, 110, 403

[17] Patterson, J., et al. 2002, PASP, 114, 721

[18] Robinson, E. L., et al. 1978, ApJ, 219, 168
[19] Sirotkin, F. \& Kim, W. 2010, ApJ, 721, 1356 doi:10.1088/0004-637X/721/2/1356

[20] Skidmore, W., et al. 2000, MNRAS, 318, 429

[21] Smak, J. 1993, Acta Astronomica, 43, 101

[22] Steeghs, D., et al. 2007, ApJ, 667, 442 doi: $10.1086 / 520702$

[23] Steele, P. R., et al. 2013, MNRAS, 429, 3492 doi:10.1093/mnras/sts620

[24] Zharikov, S., et al. 2013, A\&A, 549, A77

\section{DISCUSSION}

RAIMONDO BAPTISTA: The opening angle of the spiral structure in an accretion disk depends on the ratio of the local sound speed to a Keplerian speed. The disk region containing the spirals must be quite hot in order for the spiral will be wide open - as in your model. Is it physically consistent to get wide spiral in the disk with such low temperature such as $\sim 2000 \mathrm{~K}$.

SERGEY ZHARIKOV: Our model is a geometrical model only. Based on proposed geometrical shape and size of the disk in bounce back systems we can explain observed double-humped light curves in quiescence. Formation such spiral arm structure and physical conditions in such cool disk is not clear yet. The first approach to describe such disks was presented in paper of Cannizzo, \& Wheeler, (1984). 